\title{
Reduced baroreflex sensitivity in patients with vasovagal syncope
}

\author{
Mitro P, Simurda M, Evin L, Murin P, Muller E \\ Cardiology Clinic, Safarik University and VUSCH Kosice, Slovakia. peter.mitro@upjs.sk
}

\begin{abstract}
OBJECTIVES: The evidence is conflicting regarding the role of baroreflex in patients with vasovagal syncope. The aim of the study was to measure baroreflex sensitivity (BRS) and hemodynamic parameters during head up tilt test (HUT) with nitroglycerine stimulation.

METHODS: Nitroglycerine stimulated HUT was performed in 51 patients with the history of recurrent syncope (mean age 46 19 years, 18 men, 23 women). Cardiac output (CO), stroke volume (SV), left-ventricular ejection time (LVET) and total peripheral resistance (TPR) were assessed during HUT by volume-clamp method using a beat-to-beat photopletysmography. Spontaneous BRS sensitivity was computed using a sequential BRS calculation. RESULTS: HUT was positive after nitroglycerine administration in 28 patients and negative in 23 patients. BRS was lower at the time of syncope in HUT positive group compared to end-test values in HUT negative group $(0.54 \pm 0.27$ vs $0.72 \pm 0.35, p=0.03)$. At the time of syncope, CO was significantly lower in HUT positive patients compared to HUT negative patients $(2.6 \pm 1.4$ vs $4.3 \pm 1.4 \mathrm{l} / \mathrm{min}, \mathrm{p}<0.0001)$, similarly as SV $(34.7 \pm 14.7$ vs $49.2 \pm 19 \mathrm{ml}, p=0.005)$. LVET was significantly higher in syncopal patients $(282.27 \pm 26.2$ vs $240.5 \pm 58.8 \mathrm{~ms}$, $\mathrm{p}=0.002$ ) and TPR did not differ between two groups.

CONCLUSIONS: Reduced BRS may contribute to the development of the vasovagal syncope by inability to adequately counteract hypotension resulting from decreased cardiac output at the time of syncope (Tab. 3, Ref. 18).

Text in PDF www.elis.sk.

KEY WORDS: baroreflex, vasovagal syncope, head-up tilt test, hemodynamics.
\end{abstract}

\section{Introduction}

Vasovagal syncope is a common clinical condition with a significant impact on the quality of life of an affected individual. The pathophysiology of vasovagal syncope is only partially understood. Vasovagal syncope results from a transient cerebral hypoperfusion as a consequence of systemic hypotension. Sudden loss of sympathetic tone is considered to play a critical role in the vasodilation leading to hypotension, although this view was challenged by newer studies (1). Normal baroreflex function prevents short term oscillations in arterial blood pressure. Decrease in blood pressure leads to deactivation of baroreceptors, augmentation of sympathetic activity, inhibition of parasympathetic activity, increase in heart rate and vasoconstriction.

It could be speculated that a profound decrease in blood pressure observed in vasovagal syncope could be associated with an ineffective or only partially effective arterial baroreflex mecha-

Cardiology Clinic, Safarik University and VUSCH, Kosice, Slovakia

Address for correspondence: $\mathrm{P}$. Mitro, $\mathrm{MD}, \mathrm{PhD}$, Cardiology Clinic, Safarik University and VUSCH, Ondavska 8, SK-040 01 Kosice, Slovakia. Phone: +421.55.7891315

Acknowledgement: This work was supported by the grant VEGA [1/0479/10] and by the project "Centre for Excellent Research of Atherosclerosis and its Complications - Myocardial Infarction and Stroke", Operational Program of Research and Development financed by European Fund for Regional Development. nism. Resetting of baroreflex (reflex control is shifted to lower blood pressure levels) or reduced slope of baroreflex (reduced baroreceptor sensitivity) has been implicated in the development of vasovagal syncope (2).

The evidence is conflicting regarding the role of baroreflex in patients with vasovagal syncope. Reduced arterial baroreflex sensitivity was reported in patients suffering from vasovagal syncope in some studies $(1,3,4)$, whereas another studies did not found differences in baroreflex sensitivity in these subjects(5). In some reports, an increased baroreceptor reflex gain was observed in fainting subjects $(6,7)$.

The primary objective of the study was to assess the changes in the baroreflex sensitivity during head up tilt test with nitroglycerine stimulation and to clarify the possible role of impaired baroreflex sensitivity in the pathogenesis of vasovagal syncope. Other hemodynamic parameters were also studied during the head- up tilt test.

\section{Methods}

Fifty one patients with the history of recurrent syncope (at least 2 episodes of syncope) were included into study (mean age $46 \pm 19$ years, 18 men, 23 women). The study has been performed in accordance with the ethical standards laid down in the Declaration of Helsinki. Patients with any disease that could influence the autonomic nervous system activity were excluded from the study. All drugs affecting the autonomic nervous system were withheld for at least five half-lives. 
All patients underwent head up tilt test as a part of the standardized diagnostic evaluation of the syncope. HUT testing was performed in the morning from 9 to 12 AM after overnight fasting. HUT was performed according to Italian protocol. Patients were allowed to rest for 15 minutes in a supine position on a tilt table for hemodynamic stabilization, prior to beginning of the test. After that time, the patients were tilted at a 60 angle degree for 20 minutes or until syncope occurrence. Electronically operated tilt table with a footboard was used for the tilting of the patient. In no symptoms were observed, sublingual nitroglycerine $(0.4 \mathrm{mg}$ in spray) was administered and the test was continued for another 15 minutes without lowering the patient to supine position.

The test was considered positive when patient developed syncope or presyncope associated with hypotension and/ or bradycardia. Hypotension was defined as systolic blood pressure lower than $80 \mathrm{mmHg}$, bradycardia as heart rate bellow 50/min. The hemodynamic type of syncope was classified on the basis of modified VASIS classification (Vasovagal Syncope International Study) as mixed, cardioinhibitory and vasodepressive type.

\section{Cardiovascular parameters}

Blood pressure, heart rate and other cardiovascular parameters were assessed during HUT by continuous non- invasive photopletysmographic blood pressure monitoring device (Finometer Pro, Finapress Medical Systems, Netherlands) by means of volumeclamp method. Software package Beat Scope Easy v 020.10 (Finapress Medical System) was used for computation of stroke volume (SV), cardiac output (CO), total peripheral resistance and (TPR) and left-ventricular ejection time (LVET). Model flow method was used for beat- to-beat non-invasive hemodynamic parameter calculation. Interbeat interval (IBI) was derived from pressure pulsations as an interval between the beginning of two consecutive pulsations curves.

Baroreflex sensitivity (BRS) was computed by the same software, using a form of sequential BRS calculation (cross-sectional BRS). The correlation between time intervals and blood pressure was entered into statistics when $p=0.01$. Baroreflex sensitivity was expressed as the absolute value and as the relative value. Relative BRS was expressed as the ratio of actual BRS value to baseline BRS value (in supine position).

All cardiovascular parameters were evaluated continuously, beat-to-beat. For the statistical evaluation, parameters were divided in 5 minute periods. The arithmetical mean of all measured values in 5 minute time period was used for further statistical analysis.

\section{Statistics}

Statistical analysis was performed by the statistical software SPPS version 16.0 (SPSS Chicago, Ill, USA). Continuous variables are expressed as the mean \pm standard deviation. The unpaired Student t-test was used to test differences between the groups in parametric continuous variables. The Mann-Whitney test was used for non-pararametric continuous data. Differences in frequency distribution of specific genotypes and in other cathegorical data between HUT-positive and HUT-negative patients were tested by chi-square test and Fischer-exact test. Differences in hemodynamic parameters in various time periods during HUT were tested by analysis of variance (ANOVA) with Bonferroni comparison. Data with skewed distribution were log- transformed before entering ANOVA. P value $<0.05$ was considered statistically significant.

\section{Results}

HUT was positive in 28 patients and negative in 23 patients. There were no significant differences in clinical characteristics between HUT-positive and HUT-negative patients (Tab. 1). Baseline heart rate and blood pressure values did not differ between HUT-positive and HUT- negative patients.

Hemodynamic parameters and BRS did not differ between the two groups of patients in supine position (Tab. 1).

Blood pressure and heart rate response to tilting as well as cardiovascular parameters during tilt test are summarized in the Table 2.

In HUT positive patients, SV a CO decreased during early HUT (0-5 minutes period) in comparison to baseline values (SV 82.5 \pm 19.9 vs $64.7 \pm 18.2 \mathrm{ml}, \mathrm{p}<0.0001$, CO $5.9 \pm 1.5$ vs $5.3 \pm 1.5$ $1 /$ min, $\mathrm{p}=0.08$ ). Similar behaviour of SV and CO was observed in HUT negative patients (SV $76.9 \pm 20.9$ vs $65.3 \pm 23.3 \mathrm{ml}, \mathrm{p}<$ 0.0001 , CO $5.7 \pm 1.5$ vs $5.2 \pm 1.6 \mathrm{l} / \mathrm{min}, \mathrm{p}<0.0001)$. LVET also decreased during early HUT in HUT positive patients (328.7 \pm 21.5 vs $277.1 \pm 25.6 \mathrm{~ms}, \mathrm{p}<0.0001$ ) as well as in HUT negative patients (319.1 \pm 22 vs $279.7 \pm 24.9 \mathrm{~ms}, \mathrm{p}<0.0001$ ), with no significant difference between the two groups.

On contrary, TPR did not change significantly by assuming an upright position in HUT positive patients (1550.1 \pm 576.3 vs 1741.9 \pm 738.4 dyne.s $/ \mathrm{cm}^{5}, \mathrm{p}=0.90$ ) nor in HUT negative patients (1642.7 \pm 702 vs $1783.9 \pm 695.5$ dyne.s/cm5, $\mathrm{p}=0.37)$

BRS decreased significantly in the first 5 minutes of HUT in both group of patients (HUT positive group: $9.0 \pm 4.7$ vs $5.6 \pm 3.0$, $\mathrm{p}<0.0001$, HUT negative group $8.5 \pm 5.1$ vs $6.2 \pm 2.8$, $\mathrm{p}<0.0001$ )

Tab. 1. Clinical characteristics and cardiovascular parameters at baseline of head-up tilt test.

\begin{tabular}{lccc}
\hline & HUT + & HUT - & p \\
\hline Number of patients & 28 & 23 & \\
Men & 7 & 9 & 0.94 \\
Meanage & $48.4 \pm 17.1$ & $44.6 \pm 21$ & 0.51 \\
Number of syncopal spells & $2(1-4)$ & $3(1-5)$ & 0.11 \\
Height & $71.1 \pm 15.5$ & $67 \pm 13.5$ & 0.35 \\
Weight & $168.5 \pm 9.8$ & $166 \pm 8.4$ & 0.36 \\
SYS (mm Hg) & $142.4 \pm 16.1$ & $141.9 \pm 21.4$ & 0.92 \\
DIA (mm Hg) & $79.1 \pm 10.3$ & $80.5 \pm 15.3$ & 0.71 \\
MAP (mm Hg) & $104.3 \pm 12.1$ & $105.2 \pm 16.9$ & 0.84 \\
HR (min-1) & $71.5 \pm 10$ & $75.1 \pm 9.7$ & 0.23 \\
IBI (ms) & $864.8 \pm 119.9$ & $822 \pm 118$ & 0.24 \\
CO (l/min) & $5.9 \pm 1.5$ & $5.7 \pm 1.5$ & 0.75 \\
SV (ml) & $82.5 \pm 19.9$ & $76.9 \pm 20.9$ & 0.37 \\
TPR (dyne.s/cm ${ }^{5}$ ) & $1550.1 \pm 576.3$ & $1642.7 \pm 702$ & 0.63 \\
LVET (ms) & $328.7 \pm 21.5$ & $319.1 \pm 22$ & 0.15 \\
BRS (ms/mmHg) & $9 \pm 4.7$ & $8.5 \pm 5.1$ & 0.70 \\
BRS (ratio) & $1 \pm 0$ & $1 \pm 0$ & \\
\hline
\end{tabular}

SYS - systolic blood pressure, DBP - diastolic blood pressure, MAP - mean arterial pressure, HR - heart rate, IBI - interbeat interval, CO - cardiac output, SV - stroke volume, TPR - total peripheral resistance, LVET - left ventricular ejection time, BRS - baroreflex sensitivity 
Tab. 2. Cardiovascular parameters during passive HUT.

\begin{tabular}{|c|c|c|c|c|c|c|c|c|}
\hline \multirow[t]{2}{*}{ Time } & $0-5$ min & $0-5 \min$ & 5-10 min & 5-10 min & 10-15 min & 10-15 min & 15-20 min & 15-20 min \\
\hline & HUT + & HUT- & $\mathrm{HUT}+$ & HUT - & HUT + & HUT - & $\mathrm{HUT}+$ & HUT - \\
\hline SYS (mmHg) & $136.5 \pm 14.9$ & $138.2 \pm 21.6$ & $134.1 \pm 13.1$ & $136.3 \pm 25.5$ & $131.2 \pm 12.7$ & $137.5 \pm 31.6$ & $129.4 \pm 14.7$ & $134.1 \pm 26.5$ \\
\hline DIA (mmHg) & $81.2 \pm 9.9$ & $80.8 \pm 14.3$ & $79.6 \pm 8.8$ & $80.4 \pm 16.4$ & $79.2 \pm 9.2$ & $80.6 \pm 15.4$ & $79 \pm 10.3$ & $78.9 \pm 13.6$ \\
\hline MAP (mmHg) & $102.7 \pm 11$ & $103.3 \pm 16.3$ & $100.8 \pm 9.9$ & $102.5 \pm 19.2$ & $99.1 \pm 9.7$ & $102.6 \pm 19.4$ & $98.4 \pm 11.2$ & $100.4 \pm 16.6$ \\
\hline $\operatorname{HR}\left(\min ^{-1}\right)$ & $80.8 \pm 12.3$ & $80.2 \pm 11.7$ & $81.4 \pm 11.5$ & $80.4 \pm 12.7$ & $82.8 \pm 13.2$ & $82.5 \pm 12.4$ & $83.9 \pm 12.3$ & $82.5 \pm 12.6$ \\
\hline IBI (ms) & $761.8 \pm 114.3$ & $776.1 \pm 140.2$ & $754.3 \pm 111.2$ & $769 \pm 139.2$ & $744.5 \pm 120.7$ & $746.3 \pm 125.3$ & $731.3 \pm 110.8$ & $748.8 \pm 131.3$ \\
\hline CO (l/min) & $5.3 \pm 1.5$ & $5.2 \pm 1.6$ & $5.2 \pm 1.5$ & $5.1 \pm 1.4$ & $5 \pm 1.3$ & $5 \pm 1.4$ & $4.9 \pm 1.3$ & $4.9 \pm 1.3$ \\
\hline $\mathrm{SV}(\mathrm{ml})$ & $66 \pm 18.4$ & $66 \pm 21.2$ & $64.7 \pm 18.2$ & $65.3 \pm 23.3$ & $61.8 \pm 17.3$ & $62.3 \pm 20.7$ & $60.1 \pm 17.8$ & $61.4 \pm 19.6$ \\
\hline TPR (dyne s/cm5) & $1741.5 \pm 738.4$ & $1783.7 \pm 696.5$ & $1646.7 \pm 562.8$ & $1783.9 \pm 723.4$ & $1727.6 \pm 662.5$ & $1818.7 \pm 756.6$ & $1755.4 \pm 711.7$ & $1802.7 \pm 684.8$ \\
\hline $\operatorname{LVET}(\mathrm{ms})$ & $277.1 \pm 25.6$ & $279.7 \pm 24.9$ & $273.2 \pm 24.9$ & $274.7 \pm 25.2$ & $270.2 \pm 26.9$ & $271 \pm 27.2$ & $264 \pm 26.4$ & $272.4 \pm 25$ \\
\hline BRS (ms/mmHg) & $5.6 \pm 3$ & $6.2 \pm 2.8$ & $5.9 \pm 3.4$ & $5.6 \pm 2.6$ & $5.9 \pm 3.6$ & $5.5 \pm 2.8$ & $5.7 \pm 3.4$ & $5.5 \pm 2.8$ \\
\hline BRS (ratio) & $0.68 \pm 0.3$ & $0.8 \pm 0.22$ & $0.67 \pm 0.23$ & $0.73 \pm 0.23$ & $0.67 \pm 0.26$ & $0.71 \pm 0.23$ & $0.66 \pm 0.29$ & $0.72 \pm 0.25$ \\
\hline
\end{tabular}

- mean arterial pressure, HR - heart rate, IBI - interbeat interval, CO - cardiac output, SV - stroke volume, TPR - total peripheral resistance, LVET - left ventricular ejection time, BRS - baroreflex sensitivity

Tab. 3. Cardiovascular parameters during nitroglycerine-stimulated HUT.

\begin{tabular}{|c|c|c|c|c|c|c|}
\hline \multirow[t]{2}{*}{ Time } & 0-5 min & $0-5 \min$ & $\mathrm{p}$ & Syncope/ End & Syncope/ End & $\mathrm{p}$ \\
\hline & HUT + & HUT- & & HUT + & HUT- & \\
\hline SYS (mmHg) & $104.7 \pm 13.9$ & $119.3 \pm 19.2$ & 0.001 & $62 \pm 13.3$ & $123.5 \pm 21.5$ & $<0.0001$ \\
\hline DIA (mmHg) & $72.3 \pm 11.4$ & $78.1 \pm 14$ & 0.13 & $43.8 \pm 9.7$ & $77.3 \pm 13.8$ & $<0.0001$ \\
\hline MAP (mmHg) & $82.6 \pm 12.1$ & $92.6 \pm 15.3$ & 0.01 & $48.9 \pm 10.5$ & $95.5 \pm 20.2$ & $<0.0001$ \\
\hline $\operatorname{HR}\left(\min ^{-1}\right)$ & $104.8 \pm 17.1$ & $107.8 \pm 21.7$ & 0.61 & $76.6 \pm 30.1$ & $90.9 \pm 14.6$ & 0.05 \\
\hline IBI (ms) & $595.3 \pm 99.3$ & $582.8 \pm 133.6$ & 0.71 & $987.5 \pm 662.7$ & $656.7 \pm 155.3$ & 0.03 \\
\hline $\mathrm{CO}(\mathrm{l} / \mathrm{min})$ & $4.2 \pm 1.2$ & $4.6 \pm 1.8$ & 0.36 & $2.6 \pm 1.4$ & $4.3 \pm 1.4$ & 0.0001 \\
\hline $\mathrm{SV}(\mathrm{ml})$ & $41 \pm 13.1$ & $44.2 \pm 18.4$ & 0.51 & $34.7 \pm 14.7$ & $49.2 \pm 19$ & 0.0059 \\
\hline TPR (dyne s/cm5) & $1733.9 \pm 595.9$ & $1820.5 \pm 718.2$ & 0.66 & $2024.9 \pm 1557.3$ & $1937.1 \pm 724$ & 0.77 \\
\hline LVET (ms) & $251.1 \pm 21.7$ & $242.2 \pm 21.1$ & 0.16 & $282.2 \pm 26.2$ & $240.5 \pm 58.8$ & 0.002 \\
\hline BRS (ms/mmHg) & $4.2 \pm 2$ & $4.1 \pm 1.6$ & 0.75 & $4.5 \pm 2.4$ & $5.1 \pm 2.6$ & 0.42 \\
\hline BRS (ratio) & $0.54 \pm 0.27$ & $0.56 \pm 0.24$ & 0.40 & $0.54 \pm 0.27$ & $0.72 \pm 0.35$ & 0.03 \\
\hline
\end{tabular}

SYS - systolic blood pressure, DBP - diastolic blood pressure, MAP - mean arterial pressure, HR - heart rate, IBI - interbeat interval, CO - cardiac output, SV - stroke volume, TPR - total peripheral resistance, LVET - left ventricular ejection time, BRS - baroreflex sensitivity

There were no significant differences in hemodynamic values and BRS during passive HUT between HUT positive and HUT negative patients (Tab. 2).Similarly, no significant differences between HUT positive and HUT negative group were present during early phase (0-5 minutes) of nitroglycerine-stimulated HUT (Tab. 3).

At the time of syncope systolic BP, diastolic BP and mean BP were significantly lower in HUT positive patients when compared to HUT negative patients. HR values did not differ significantly, but a trend to lower values in HUT positive group was observed and the interbeat interval was significantly longer in syncopal patients (Tab. 3)

CO significantly decreased at the time of syncope when compared to the initial phase of nitroglycerine stimulated HUT (4.2 \pm 1.2 vs $2.6 \pm 1.4, \mathrm{p}<0.0001$ ) and was significantly lower in HUT positive patients compared to HUT negative patients ( $2.6 \pm 1.4$ vs $4.3 \pm 1.4, \mathrm{p}<0.0001)$. Similarly, SV decreased and was significantly lower in HUT positive patients at the time of syncope (34.7 \pm 14.7 vs $49.2 \pm 19$, $\mathrm{p}=0.005)$

LVET was significantly higher in syncopal patients (282.27 \pm 26.2 vs $240.5 \pm 58.8 \mathrm{~ms}, \mathrm{p}=0.002$ ). TPR at the time of syncope did not differ significantly between the two groups of patients ( $\mathrm{p}$ $=0.77$ ) (Tab. 3).

Absolute values of BRS were lower in syncopal patients, but the difference was not significant. However, the relative BRS value
(BRS ratio) was significantly lower at the time of syncope in HUT positive group when compared to end-test values in HUT negative group $(0.54 \pm 0.27$ vs $0.72 \pm 0.35, \mathrm{p}=0.03)$.

\section{Discussion}

The main finding of our study is that in the subjects with recurrent syncope and positive nitroglycerine stimulated HUT, we observed a decreased baroreflex sensitivity at the time of syncope. From the hemodynamic point of view, we observed decreased SV and CO during HUT-induced syncope. This is in agreement with previously published results. Decrease in cardiac output is considered the main hemodynamic mechanism responsible for the development of vasovagal reaction during nitroglycerine stimulated HUT (8). Fu et al reported that a decrease in CO is invariably present in healthy persons during vasovagal reaction, while a decrease in TPR was not present in about one third of subjects (9). Indeed, we did not observe significant differences in TPR between patients with positive and negative HUT, despite a lower blood pressure in HUT-positive subjects. Thus, it seems that hypotension in our patients was a consequence of decreased cardiac output rather than vasodilation.

LVET is influenced by various factors, one of them is myocardial contractility. Increased myocardial contractility leads to the shortening of LVET. In our study, patients with positive HUT 
showed a higher LVET at the time of syncope than HUT-negative patients. This finding can be interpreted as a decreased myocardial contractility at the time of syncope, which could be a consequence of decreased sympathetic stimulation at the time of syncope. Similar findings were reported by Couceiro et al (10). They showed a bi-phasic behaviour of LVET during HUT in patients with vasovagal syncope. In the first period of HUT, a decrease in LVET reflecting an increased myocardial contractility was present as a compensatory mechanism responding to venous pooling. In the second half of HUT, failure of this compensation was seen with concomitant reduction of sympathetic activity and simultaneous LVET increase.

Investigation of baroreflex sensitivity in patients with vasovagal syncope showed controversial results. This may depend on the different methods used in published studies. Besides stimulusdependent methods (phenylephrine infusion, Valsalva maneuver, neck chamber technique), an analysis of spontaneous baroreflex sensitivity is available. Two principal methods for the analysis of spontaneous baroreflex sensitivity were proposed so far: the sequence method and spectral method (11). In our study, we used a sequence method originally described by Parati, which is based on identification of at least three consecutive beats, in which progressive changes in systolic blood pressure are followed by progressive changes in RR interval (12).

Advantage of spontaneous baroreflex sensitivity evaluation is a better reproducibility in comparison to stimulus dependent methods and the fact that it can be used to evaluate short time periods and in this way it is possible to measure BRS during different phases of HUT. It is less time consuming and it is not operator-dependent (11).

Iacoviello et al used a sequence method in evaluation of the spontaneous baroreflex sensitivity during nitroglycerine-stimulated HUT. In patients with syncope, they reported lower baroreflex sensitivity values immediately before syncope onset in comparison to HUT negative patients at the end of the HUT (13). In our study, we used the same methodology of HUT and baroreflex assessment. Our observations are similar with the results of above mentioned group, although only relative BRS values were significantly lower in our patients with positive HUT and the difference in absolute BRS did not reach the statistical significance.

The same group of authors in the subsequent study showed that reduced baroreflex sensitivity during HUT was an independent predictor of syncope recurrence. This finding supports the hypothesis that impaired baroreflex sensitivity may play an important role in the pathogenesis of vasovagal syncope (14).

Reasons for baroreceptor reflex dysfunction in vasovagal syncope are not known. It was suggested that changes in respiratory pattern preceding full-blown vasovagal syncope may play an important role (15). Reciprocal influences exist between respiratory and cardiovascular activity. Hyperventilation in the last three minutes preceding bradycardia and hypotension was reported (16) . Hyperventilation results in hypocapnia and cerebral vasoconstriction in the chemoreflex areas of the brain stem. This leads to an increased chemoreceptor gain, which in turn may decrease baroreflex sensitivity (17).
An alternative explanation for baroreflex dysfunction in vasovagal syncope is based on primary dysfunction of vasculature, leading to its inability to maintain the appropriate vasomotor tone. Bechir et al (2) calculated spontaneous cardiac baroreflex sensitivity in patients with vasovagal syncope using a spectral analysis technique. In addition, he directly measured sympathetic nervous activity by direct microneurography recordings from peroneal nerve. Lower body negative pressure (LBNP) was used to simulate orthostatic stress. They showed a decreased baroreflex sensitivity both at baseline and during LBNP. Resting muscle sympathetic nerve activity (MSA) as assessed by microneurography was increased at baseline. During various degrees of LBNP, a blunted activation of MSA was present. This suggests the dysfunction in vascular bed, which generates an increased sympathetic activity in order to maintain preload in patients with vasovagal syncope and resets baroreflex sensitivity. Baroreflex subsequently operates at lower levels of blood pressure. This may explain a decreased resting BRS. On the other hand, the ability to increase sympathetic activity during condition of increased demands (i.e. orthostasis) is attenuated.

Dysfunctional baroreceptor reflex was also reported in HUT positive patients when moving from upright to supine position at the end of tilt test. Normal response to sudden volume loading of central circulation is bradycardia and diminished vasoconstriction due to sympathetic inhibition. On contrary to an expected response, acceleration of heart rate and increase in blood pressure was reported as a manifestation of decreased baroreflex activity in position change from upright to supine position (18).

Limitations of our study are associated with non-invasive assessment of beat-to beat hemodynamic by Model flow methods. The absolute values of these parameters differ from invasively measured parameters. On the other hand, identifying of trend and changes is more important in our studies than measuring the absolute values. Another limitation is the fact, that patient study group was selected by excluding patients with organic heart disease, which could influence an autonomic balance, so the result of our study are not applicable on the whole group of patient with vasovagal syncope.

In conclusion, reduced baroreflex sensitivity may contribute to the pathogenesis of vasovagal syncope by inability to adequately counteract hypotension resulting from decreased cardiac output at the time of syncope.

\section{References}

1. Vaddadi G, Esler MD, Dawood T, Lambert E. Persistence of muscle sympathetic nerve activity during vasovagal syncope. Eur Heart J 2010; 31: 2027-2033.

2. Bechir M, Binggeli C, Corti R et al. Dysfunctional baroreflex regulation of sympathetic nerve activity in patients with vasovagal syncope. Circulation 2003; 107: 1620-1625.

3. Mosqueda-Garcia R, Furlan R, Fernandez-Violante R et al. Sympathetic and baroreceptor reflex function in neurally mediated syncope evoked by tilt. J Clin Invest 1997; 99: 2736-2744. 


\section{2-586}

4. Morillo CA, Eckberg DL, Ellenbogen KA et al. Vagal and sympathetic mechanisms in patients with orthostatic vasovagal syncope. Circulation 1997; 96: 2509-2513.

5. Thomson HL, Wright K, Frenneaux M. Baroreflex sensitivity in patients with vasovagal syncope. Circulation 1997; 95: 395-400.

6. Vaddadi G, Corcoran SJ, Esler M. Management strategies for recurrent vasovagal syncope. Intern Med J 2010; 40: 554-560.

7. Chan MY, Lin M, Lucas $\mathbf{J}$ et al. Plasma proteomics of patients with non-valvular atrial fibrillation on chronic anti-coagulation with warfarin or a direct factor Xa inhibitor. Thromb Haemost 2012; 108: 1180-1191.

8. Verheyden B, Liu J, van Dijk $\mathbf{N}$ et al. Steep fall in cardiac output is main determinant of hypotension during drug-free and nitroglycerine-induced orthostatic vasovagal syncope. Heart Rhythm 2008; 5: 1695-1701.

9. Fu Q, Verheyden B, Wieling W, Levine BD. Cardiac output and sympathetic vasoconstrictor responses during upright tilt to presyncope in healthy humans. J Physiol 2012; 590: 1839-1848.

10. Couceiro R, Carvalho P, Paiva RP et al. Characterization of surrogate parameters for blood pressure regulation in neurally-mediated syncope. Conf Proc IEEE Eng Med Biol Soc 2013; 2013: 5381-5385.

11. La Rovere MT, Pinna GD, Raczak G. Baroreflex sensitivity: measurement and clinical implications. Ann Noninvasive Electrocardiol 2008; 13: 191-207.
12. Parati G, Di Rienzo M, Bertinieri G et al. Evaluation of the baroreceptor-heart rate reflex by 24-hour intra-arterial blood pressure monitoring in humans. Hypertension 1988; 12: 214-222.

13. Iacoviello M, Guida P, Forleo C, Sorrentino S, D’Alonzo L, Favale S. Impaired arterial baroreflex function before nitrate-induced vasovagal syncope during head-up tilt test. Europace 2008; 10: 1170-1175.

14. Iacoviello M, Forleo C, Guida $\mathbf{P}$ et al. Independent role of reduced arterial baroreflex sensitivity during head-up tilt testing in predicting vasovagal syncope recurrence. Europace 2010; 12: 1149-1155.

15. Lagi A, Cencetti S, Cartei A. What Happens before Syncope? Study of the Time Frame Preceding Vasovagal Syncope. ISRN Cardiol 2011; 2011: 659787.

16. Kurbaan AS, Erickson M, Petersen ME et al. Respiratory changes in vasovagal syncope. J Cardiovasc Electrophysiol 2000; 11: 607-611.

17. Somers VK, Mark AL, Abboud FM. Interaction of baroreceptor and chemoreceptor reflex control of sympathetic nerve activity in normal humans. J Clin Invest 1991; 87: 1953-1957.

18. Samniah N, Sakaguchi S, Ermis C, Lurie KG, Benditt DG. Transient modification of baroreceptor response during tilt-induced vasovagal syncope. Europace 2004; 6: 48-54. 